\section{alettsomian alpectures}

on

\section{THE SURGICAL DISEASES OF CHILIREN.}

\author{
DELIVERED BEFORE TIIE MEDICAL SOCIETY \\ OF LONDON.
}

BY

T H O M S B R Y N T, F.R.C.S., ASSISTANT-SERGEON TO GUY'S HOSPITAL.

\section{Lecture I. (Continued.) \\ Malformatioxs of the Anus axd Lower Bowes.}

Havivg thus far occupied your attention by dwelling for some length upon the congenital deficiencies which are to be observed at the upper orifice of the mucous or digestive canal, I propose to pass downwards to the other or terminal end, and to discuss the subject of imperforate anus or occluded rectum; and to attempt, from general as well as from personal experience, to bring before you some practical points, which may be of service in the treatment of this iuteresting and difficult class of cases. These malformations of the rectum may be divided into three great sections.

1. The simplest form, which includes the cases in which the orifice of the anus is completely closed, the rectum being partially or wholly deficient.

2. The more complicated class of cases, in which the anus exists in its natural condition, but opens into a cul-de-sac; the rectum being partially or wholly deficient.

3. Cases of imperforate anus, the rectum opening either into the vagina or urethra, or in some other abnormal position.

Under one or other of these headings can nearly all the cases of this congenital defect be readily classed.

To Mr. Curling's exhaustive paper, published in the forty-third volume of the Medico-Chirurgical Transactions, we are unquestionably indebted for the bulk of our information upon this subject; and as his description of these deformities with their assigned causes is so good, I may be pardoned for quoting them at some length.

It will be observed (says Mr. Curling) that the classification of these imperfections is founded on states which can generally be recognised during life. Unfortunately, the condition of the terminal portion of the intestinal canal, and its relations to the parts around, cannot be predicated with any certainty. In cases of imperforate anus, or of anus opening into a $c u /-d e-s a c$, the intestinal canal may terminate in a blind pouch at the brim of the pelvis, the rectum being wholly wanting; or an imperfect rectum may form a short sac, descending to the floor of the pelvis, or as low as the neck of the bladder in the male, or the commencement of the vagina in the female.

It is known that the anal portion of the bowel is developed distinctly from the upper portion, and that the two afterwards approximate and unite, the diaphragm or septum disappearing by intestinal absorption. A failure in this process is the cause of the second form of congenital imperfection.
The cases of imperforate anus in which the rectum communicates with the urethra or vagina depend on the original existence of a cloaca, the malformation being due to an incomplete separation during fœtal life. These conditions are the result of an arrest of development at different stages. 'ihe blind pouch, in which the intestinal canal terminates, is sometimes connected to the anal integument, or to the anal cul-de-sac, by a cord prolonged from the bowel above. These cases are not, like the preceding, the result of a non-formation of the rectum; but are produced by an obliteration of the bowel, which was originally well-formed; the obliteration being a pathological change, due, probably, to inflammation which had existed during intrauterine life.

Cases are quoted by Curling from M. Goyrand and Friedberg, where the muscular tissue of the intestine was clearly traced, which point with considerable force to the accuracy of these views.

It is, therefore, with a view of determining the practice which should be employed in these different varieties of malformed anus and rectum, that I now purpose to enter upon the consideration of this subject.

Treatment of the First Class of Cases. In the first class of cases in which the anus is closed or absent altogether, there would not be much difficulty in arriving at a correct conclusion as to the practice which should be followed, if it were possible for the surgeon to learn with accuracy by any examination the true position of the bowel, the terminal end of which is evidently malformed. It may be that the anus is the only part in fault, and that the bowel is natural as regards position, its orifice being alone closed. In these fortunate instances, there is not grenerally any great difficulty in determining the true condition of the parts; for a distinct bulging of the distended rectum will, in all probability, be readily felt on a careful manual examination; and in many cases the eye will at once detect the fact that a thin membrane alone obstructs the orifice. In such instances as these, there can be no doubt as to the practice which is to be pursued; a cautious incision over the spot in which the anus exists, or should exist, at once suggesting itself to the mind of the refiective surgeon. But this practice must be carried out with considerable care, and the finger should be the pilot. The incision must be carried backwards towards the sacrum, and not forwards towards the urethra or vagina, and it should be tolerably free; and under such circumstances, a free discharge of the retained meconium will in these simple cases be generally obtained.

In the more obscure class of cases of occluded anus, in which no such bulging of the bowel is to be seen or even felt, the practice which is to be carricd out is not so certain, at any rate in its results; this uncertainty being due to the fact of its being perfectly impossible for the surgeon to form any opinion as to the true position of the malformed bowel. The rectum may be in its natural place, or it may not; it may terminate at the brim of the pelvis on the left side, or on the right, or it may not exist; and, under these diverse circumstances, the success of an exploratory operation in the perinaum cannot but be uncertain; although I believe it to be justifiable if carried out with care.

The plunging of a large trocar and cannula into 
the pelvis with the vain hope of puncturing the distended intestine, is a practice which must be unhesitatingly condemned. It is unscientific on principle and unsuccessful in practice. It is injurious to surgery, as it is not based on a correct foundation; and injurious to our patients, as it is most fatal in its effects. Nevertheless, I believe that an exploratory operation in the perinæum, if conducted with care, is the best practice which can be primarily carried out.

An incision should be made in the same region as pointed out in the last class of cases-that is, in the position in which the anus should be, when naturally placed; and then, guided by the finger in the direction backwards and upwards, keeping the sacrum as the guide, the surgeon may fairly make an attempt to reach the bowel.

The tension of the distended intestine is to be readily recognised, particularly when it has been once felt; and, having opened it, the operator should use all fair endeavours to draw down the intestine to the margin of the external opening, and to fasten it to the integument whenever it is found possible. But the hope of being able to succeed in doing this is not great, although the adrantages which are to be acquired by success warrant the attempt.

The bowel, in the majority of cases, however, terminates at some distance from the surface. Mr. Curling states that " it is rarely found at a less distance than an inch from the perinæum"; and when we consider the shallowness of the child's pelvis, this depth is very great; the fact fairly indicating to us the probability that the rectum terminates in the majority of cases at the brim of the pelvis, and seldom dips down into it.

Out of twenty-six cases of this variety, which Mr. Curling has tabulated, the bowel was opened in fourteen; and in the remaining twelve, failure followed the attempt. Of the fourteen cases in which the bowel was opened, five only proved successful; whilst of the twelve cases in which the attempt failed, the bowel was subsequently opened either in the loin or groin in ten cases, and three of these died; the remaining two dying without anything further being done.

It must, however, be added that the great fatality of the instances in which the bowel was reached is to be explained by the rashness with which the exploratory operation was performed; a blind perforation of the parts with a trocar, with the vague hope of securing success, forbidding, in many cases, any prospect of recovery; the post mortem examination too often revealing the fact that the bladder, uterus, and peritoneum had been freely punctured, and that death was the result of the secondary peritoneal inflammation.

These figures prove to us the danger of the perineal exploratory operation, and shew that, unless it be undertiken with great care, more harm than good will probably ensue; nevertheless the propriety of making the attempt cannot be questioned, although it must be conducted with extreme caution.

Treatrnent of the Second Class of Cases. In the second or more complicated class of cases, in which the anus exists in its natural condition, but opens into a cul-de-sac, the rectum being partially or wholly deficient, the uncertainty of being able to form any opinion as to the true position of the lower bowel, renders any operative measure as hazardous, as it has been shown to be in the former division; although it can be proved that, when the attempt to relieve has proved successful, a large proportion of the cases terminate well.

The upper portion of bowel may be in contact with the extremity or side of the cul-de-sac, or it may be some distance from it; but there certainly appears to be a stronger probability that the two extremities of the bowel will be found nearer in position in these cases, than in the former class in which no $c u l-d e-s a c$ exists.

The upper bowel, in almost every instance, terminates somewhere at the pelvic brim; although it appears to be quite a matter of chance whether its terminal end lie to the right or left side of the pelvis, or on the sacral promontory ; and as the cillde-sac is evidently an attempt of the lower part to meet the upper portion of the bowel, the distance between the two ends is not generally very great.

Under such circumstances, an exploratory operation at the extremity and posterior part of the culde-sac appears to be justifiable on principle; and, when conducted with judgment, it has also been practically proved of value.

Mr. Curling gives us thirty-one examples of this class of cases. In twenty-seven, an attempt was made to reach the bowel, and in sixteen the attempt was followed by success, and ten of these subsequently recovered. In eleven instances, failure followed the attempt; and, although other measures were carried out, all died. In four cases, colotomy was alone employed; three in the groin with success, one in the loin which died.

In these cases, it would therefore also appear that an exploratory operation at the part is perfectly justifiable when conducted with caution; and that, if such should fail, colotomy is to be performed.

The exploratory operation must, however, be cautiously carried out; if a bulging at any spot can be detected, the attention of the surgeon should be directed to this part. An exploratory puncture may be made; a fine trocar and cannula, I believe, being as good an instrument as any that can be employed. It must be introduced carefully, and in the direction indicated, avoiding the anterior portion of the passage, and directing the point backwards and upwards. If any evidence can, in this way, be obtained of the bowel being perforated, the trocar may be taken out, and a grooved probe introduced in its stead, over which the cannula can be readily removed. The opening may subsequently be freely enlarged by means of a bistoury, and the divided bowel brought down and stitched to the cul-de-sac when found possible.

In the following case, the practice thus briefly sketched was carried out; and if a successful result forms a recommendation for the practice, it can in this instance be adduced.

CASE I. Edward B., aged 10 days, was brought to me at Guy's Hospital on January 15th, 1862, from Lower Norwood. He had not passed any motion since his birth, and had vomited freely. The child's general condition, however, appeared to be good.

On examining the part, a good anus was readily observed, and this led into a canal, which was about one inch long, ending by a cul-de-sac. The extremity of this was smooth, firm, and unyielding, and no evidence of any bulging of the bowel could be detected. 
An exploratory operation was, however, deemed ad. visable; and I therefore introduced a fine exploring trocar and cannula, no larger than a fine probe, into the upper and posterior part of the cul-ie-sec, and, using considerable care in the exploration, came agaiust an elastic, firm body; this was punctured, and evidence of faces was tolerably clear; a grooved probe was then passed through the cannula, and over it the cannula was removed; by means of a curred bistoury, the opening was subsequently enlarged, and about an ounce of meconium came away. In half an hour afterwards, however, the bowels were freely relieved ; the faces being of a semisolid consistence.

For domestic reasons, the mother was unable to stay at the hospital, and the child was therefore taken home. On the second day, the child appeared to be well in every respect; it had apparently been free from all abdominal pain; the motions had passed readily, and were of a healthy character; the abdomen was small, and all vomiting had ceased since the operation. The finger could be passed readily into the bowel, although a tight ring was at once obscrved. This digital dilatation was directed to be maintained daily; and $M r$. Baker, the medical adviser of the family, kindly undertook to watch the case.

On March 7 th, I had a letter from Mr. Baker, which contained a favourable report. Everything was going on well. The dilatation of the opening was persevered in, and no contraction had apparently taken place. The child's health was also good. Mr. Baker, however, added his fear that, unless constant dilatation was persevered in, contraction would follow.

The last report was on Dec. 10th; after which, the child having left the neighbourhood, Mr. Baker could give no intelligence.

In this instance, the success which followed the measures which were employed appears to me perfectly to justify the practice; and I do not think that a careful exploratory puncture by mears of a fine trocar and cannula can be looked upon as less scientific or valuable than a cautious exploratory incision with a bistoury. The evils of a reckless and incautious introduction of a trocar and cannula cannot be too strongly condemned; but the same recklessness in the operator would attend the exploratory incision by means of a scalpel, and an equally bad result would probably follow.

To attain permanent success, however, in these cases, considerable attention is afterwards rigidly demanded. Repeated dilatation of the perforated bowel is absolutely essential to maintain its patency; for otherwise, like all artificial openings, its subsequent contraction will take place, with all the evils and fatal results of an ordinary stricture.

It will be gathered, however, from what has just been stated, that, in a large proportion of the cases of imperforate anus and obstructed rectum, a successful exploratory operation in the ano-perinaal region is not always to be recorded ; for, in nearly half the recorded cases, or in 43 per cent., failure followed the attempt; and, as a consequence, the surgeon has in like instances to consider the important question as to the best means for affording any further prospect of relief.

Two operations suggest themselves for consideration : first, the opening of the bowel in the left loin, known as Callisen's or Amussat's operation; and, secondly, the one known as Littre's, or the opening of the bowel in the left groin.

The former operation, known as Callisen's, Amussat's, or the lumbar operation, is not one which offers many advantages. The irregularities in the position of the colon in the left loin, even in a healthy subject, become still greater in these cases of maldevelopment; and to the operating surgeon, they are, therefore, sources of considerable difficulty. The depth of the colon, when naturally placed in the young child ; the probability of the kidney occupying the position of the colon; and the prospects of finding the bowel empty at the time of operation, - are also additional arguments against its performance; but the strongest of all reasons remains to be repeated, and that is the bad success which has followed its adortion; for, in seven instances in which it has been performed, five died. The operation is, therefore, one of uncertainty, difficulty, and danger; and these points become of greater power when it can be shewn that Littre's, or the inguinal operation, is attended with greater certainty, less difficulty, and a better success.

I need hardly repeat, that Littre's operation consists of the opening of the sigmoid flexure in the left groin. The integuments in this region are thin and readily divided; the bowel lies immediately beneath, and can without difficulty be opened; and it can be hardly said that the inconveniences of an artificial anus in this region are greater than they are in the lumbar operation. In these respects, therefore, the inguinal operation has the preference; and the greater success with which the practice has been attended tends to support it still more. Out of fourteen instances in which it has been performed, nine recovered; and it will be remembered that two only out of seven recovered after the lumbar operation.

It can, therefore, I think, be fairly stated that the inguinal operation is the one which the weight of evidence tends to support; and that, in the cases in which a careful exploratory operation in the anoperinaal region has failed to afford relief, such may be looked for with some confidence through these means.

\section{[To be continued.]}

Parliamentary Votes for Hospitals. The following votes have been taken in the House of Commons. $£ 1,600$ for the Lock Hospital; $\$ 700$ for the Rotunda Lying-in Hospital ; 200 for the Coombe Lying-in Hospital ; $\{1,600$ for the House of Industry ; $\$ 1,500$ for the Cork Street Fever Hospital ; $\mathfrak{L}(600$ for the Meath Hospital; $\mathfrak{i} 100$ for St. Nark's Ophthalmic Hospital; $\mathfrak{\&} 1,300$ for Dr. Steevens's Hospital; $22 \pm 5$ for the Loard of Superintendence and Dublin Hospitals.

Extraondinary Deatir. A warder of the Bagne at Toulon, has just met his death in the following manner : He was amusing himself, while off duty, with fishing in the dock, when, having caught a fish about seven inches long and two broad, and not knowing where to place it, while baiting his hook conceived the idea of holding it between his teeth. 'The fish struggling in the convul. sions of death, ended by slipping its head first into the mouth and thence owing to the viscous matter, with which the scales were covered, down his throat, completely filling up the cavity. The man rushed about for aid, but soon dropped dead from suffocation. 